Network Working Goup

Request for Comments: 2706

D. Eastlake

Category: Informational

T. Goldstein

Brodia

October 1999

ECML v1: Field Names for E-Commerce

Status of this Memo

This memo provides information for the Internet community. It does not specify an Internet standard of any kind. Distribution of this memo is unlimited.

Copyright Notice

Copyright (C) The Internet Society (1999). All Rights Reserved.

IESG Note

This document is the output of a vendor consortium, and is not the output of an IETF Working Group. Implementors of this specification are warned that this data model is heavily biased toward conventions used in the United States, and the English language. As such it is unlikely to be suitable for international or multilingual use in the global Internet.

Abstract

Customers are frequently required to enter substantial amounts of information at an Internet merchant site in order to complete a purchase or other transaction, especially the first time they go there. A standard set of information fields is defined as the first version of an Electronic Commerce Modeling Language (ECML) so that this task can be more easily automated, for example by wallet software that could fill in fields. Even for the manual data entry case, customers will be less confused by varying merchant sites if a substantial number adopt these standard fields. 


\title{
Acknowledgements
}

The following persons, in alphabetic order, contributed substantially to the material herein:

\author{
George Burne, Trintech \\ Joe Coco, Microsoft \\ Kevin Weller, Visa
}

Table of Contents

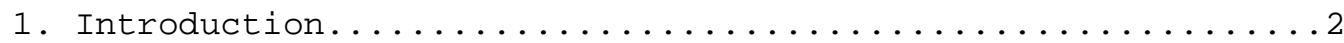

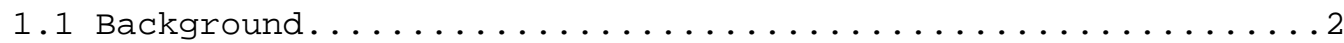

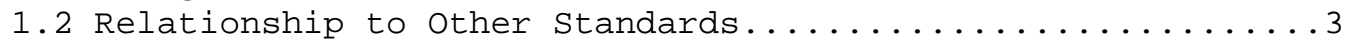

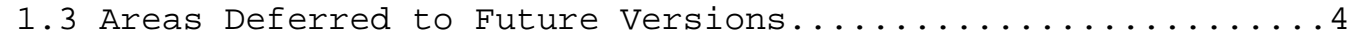

2. Using The Fields..........................

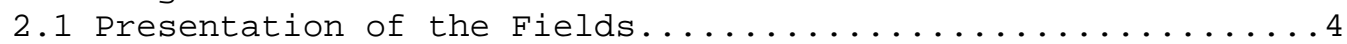

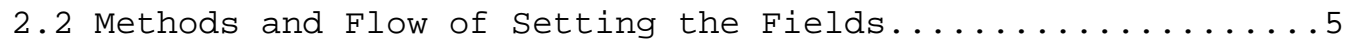

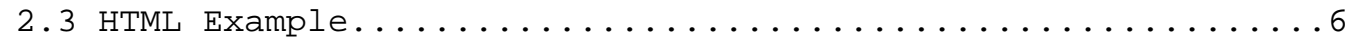

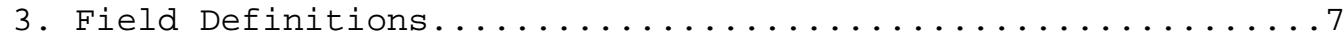

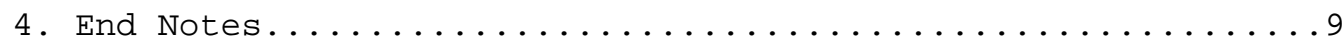

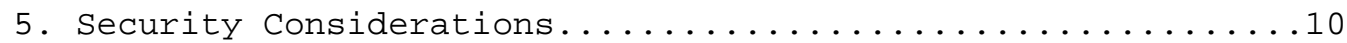

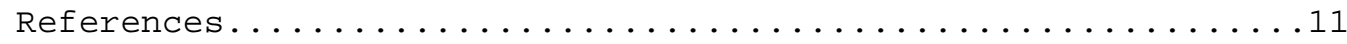

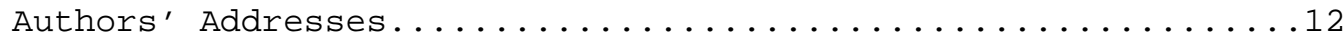

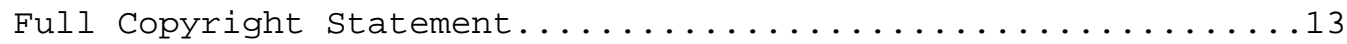

1. Introduction

\subsection{Background}

Today, numerous merchants are successfully conducting business on the Internet using HTML-based forms. The data formats used in these forms varies considerably from one merchant to another. End-users find the diversity confusing and the process of manually filling in these forms to be tedious. The result is that many merchant forms, reportedly around two thirds, are abandoned during the fill in process.

Software tools called electronic wallets can help this situation. A digital wallet is an application or service that assists consumers in conducting online transactions by allowing them to store billing, shipping, payment, and preference information and to use this information to automatically complete merchant interactions. This greatly simplifies the check-out process and minimizes the need for a consumer to complete a merchant's form every time. Digital wallets that fill forms have been successfully built into browsers, as helper 
applications to browsers, as stand-alone applications, as browser plug-ins, and as server-based applications. But the proliferation of electronic wallets has been hampered by the lack of standards.

ECML (Electronic Commerce Modeling Language, <www.ecml.org>) Version 1 provides a set of simple guidelines for web merchants that will enable electronic wallets from multiple vendors to fill in their web forms. The end-result is that more consumers will find shopping on the web to be easy and compelling.

The set of fields documented herein was developed by the Wallet/Merchant Standards Alliance (www.ecml.org) which now includes, in alphabetic order, the following:

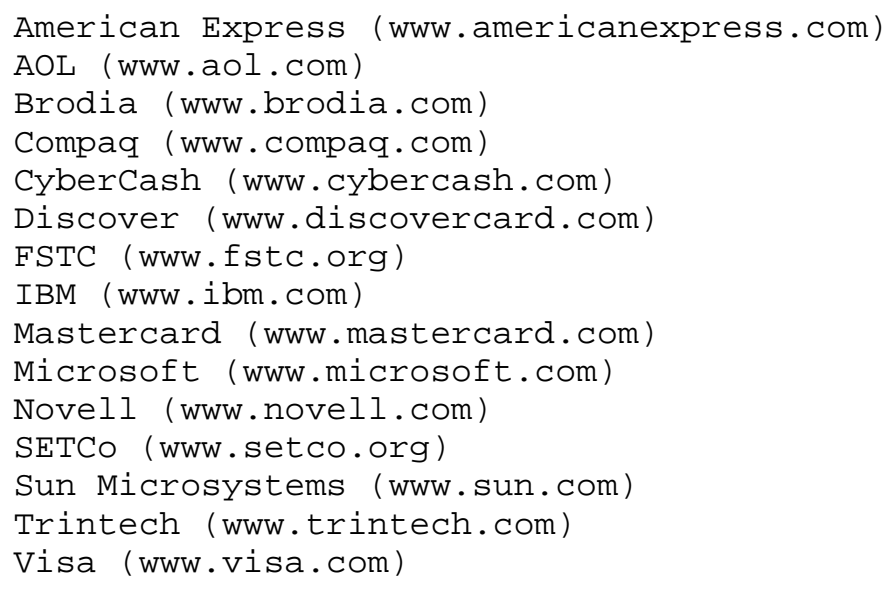

The fields are derived from and consistent with the W3C P3P base data schema at

<http://www.w3.org/TR/WD-P3P/basedata.html>.

\subsection{Relationship to other Standards}

ECML Version 1 is not a replacement or alternative to SSL/TLS [RFC 2246], SET [SET], XML [XML], or IOTP [IOTP]. These are important standards that provide functionality such as non-repudiatable transactions, automatable payment scheme selection, and smart card support.

ECML may be used with any payment mechanism. It simply allows a merchant to publish consistent simple web forms.

Multiple wallets and multiple merchants plan to interoperably support ECML. This is an open standard. ECML is designed to be simple. 
Version 1 of the project adds no new technology to the web. A merchant can adopt ECML and gain the support of these multiple Wallets by making very simple changes to the HTML pages that they currently use to support their customers. Use of ECML requires no license.

\subsection{Areas Deferred to Future Versions}

Standardization of information fields transmitted from the merchant to the consumer, considerations for business purchasing cards, noncard payment mechanisms, wallet activation, privacy related mechanisms, additional payment mechanisms, and any sort of "negotiation" were among the areas deferred to consideration in future versions. Hidden or other special fields were minimized. The primary target was North American consumer to merchant electronic commerce.

2. Using The Fields

To conform to this document, the field names shall be as listed in section 3 below. Note: this does not impose any restriction on the user visible labeling of fields, just on their names as used in communication with the merchant.

\subsection{Presentation of the Fields}

There is no necessary implication as to the order or manner of presentation. Some merchants may wish to ask for more information, some less by omitting fields. Some merchants may ask for the information they want in one HTML form on one web page, others may ask for parts of the information at different times on different pages. For example, it is common to ask for "ship to" information earlier, so shipping cost can be computed, before the payment method information. Some merchants may require that all the information they request be provided while other make much information optional, etc.

There is no way with version 1 of ECML to indicate what fields the merchant considers mandatory. From the point of view of customer software, all fields are optional to complete. However, the merchant may give an error or re-present a request for information if some field it requires is not completed, just as it may if a field is completed in a manner it considers erroneous. 


\subsection{Methods and Flow of Setting the Fields}

There are a variety of methods of communication possible between the customer and the merchant by which the merchant can indicate what fields they want that the consumer can provide. Probably the easiest to use for currently deployed software is as fields in an HTML [HTML4.0] form. Other possibilities are to use the W3C P3P protocol or the IOTP Authenticate transaction [IOTP].

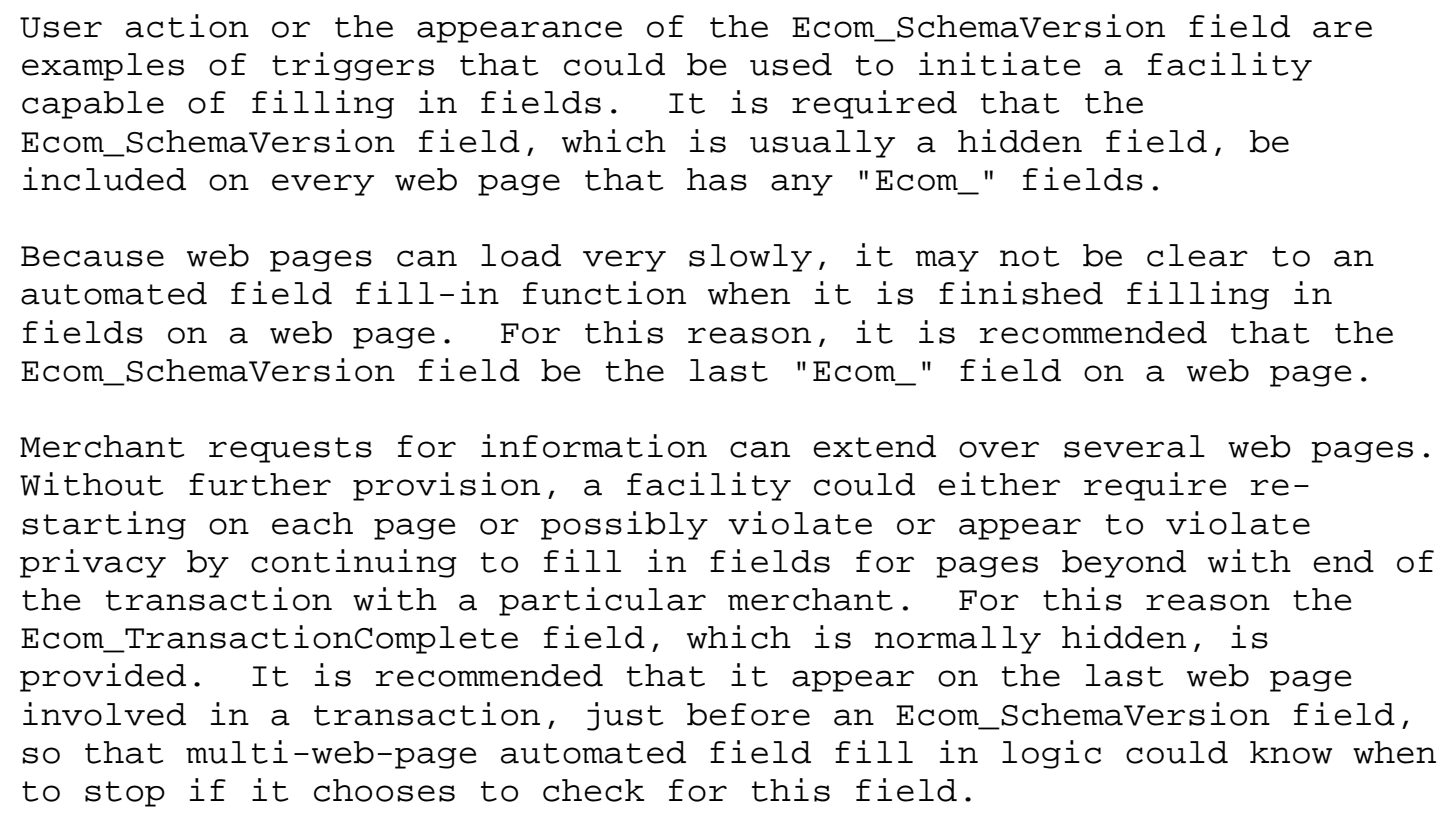




\subsection{HTML Example}

An example in HTML might be as follows:

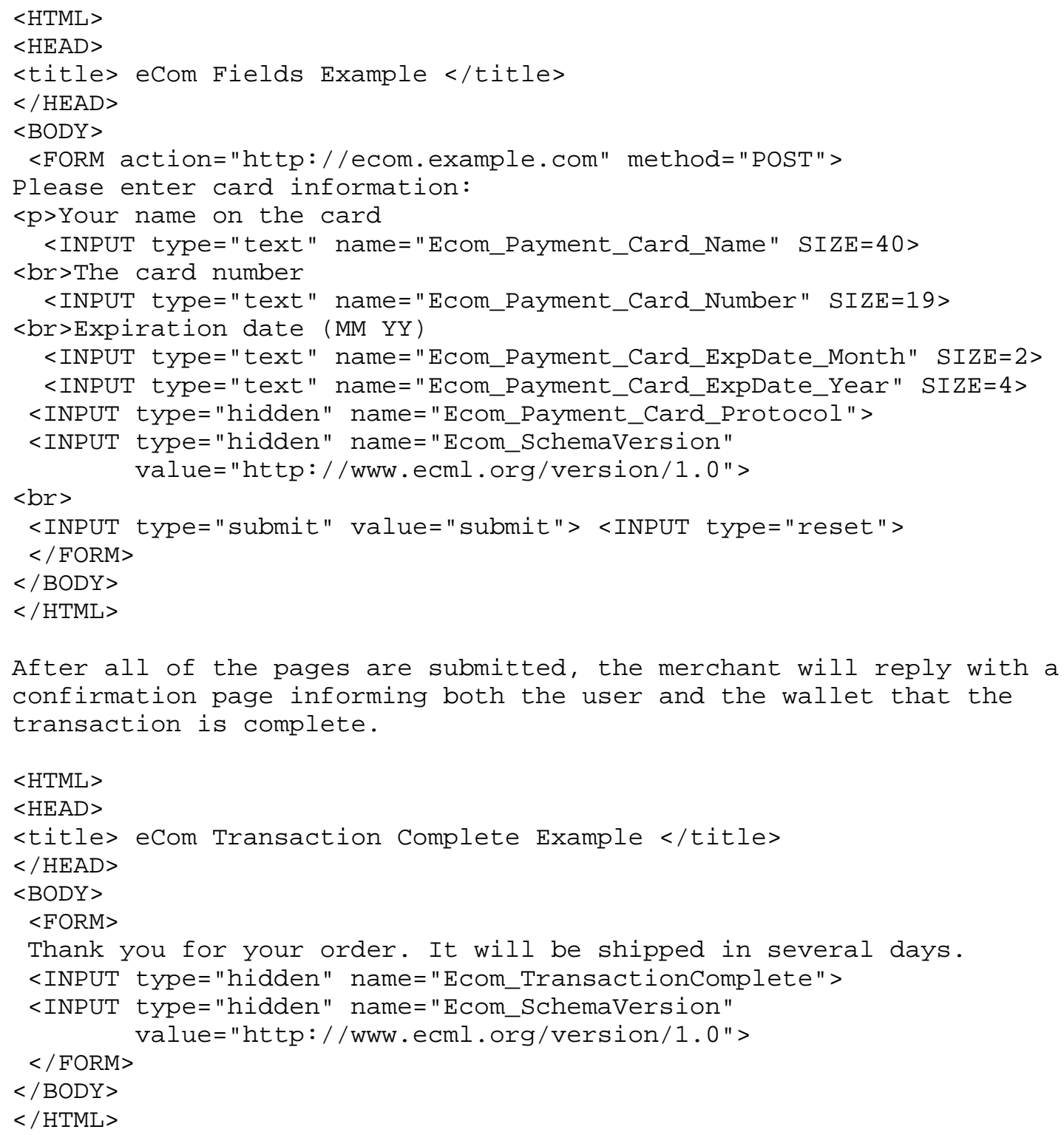




\section{Field Definitions}

The fields are listed below along with the minimum data entry size to allow. Note that these fields are hierarchically organized as indicated by the embedded underscore ("_") characters. Appropriate consumer to merchant transmission mechanisms may use this to request and send aggregates, such as Ecom_Payment_Card_ExpDate to encompass all the date components or Ecom_ShipTo to encompass all the ship to components that the consumer is willing to provide. The marshalling and unmarshalling of the components of such aggregates depends on the data transfer protocol used.

IMPORTANT NOTE: "MIN" in the table below is the MINIMUM DATA SIZE TO ALLOW FOR ON DATA ENTRY. It is NOT the minimum size for valid contents of the field and merchant software should, in most cases, be prepared to receive a longer or shorter value. Merchant dealing with areas where, for example, the state/province name or phone number is longer than the "Min" given below must obviously permit longer data entry. In some cases, however, there is a maximum size that makes sense and where this is the case, it is documented in a Note for the field.

FIELD

ship to title
ship to first name
ship to middle name
ship to last name
ship to name suffix
ship to street linel
ship to street line2
ship to street line3
ship to city
ship to state/province
ship to zip/postal code
ship to country
ship to phone
ship to email
bill to title
bill to first name
bill to middle name
bill to last name
bill to name suffix
bill to street linel
bill to street line2
bill to street line3
bill to city

NAME

Ecom_ShipTo_Postal_Name_Prefix Ecom_ShipTo_Postal_Name_First Ecom_ShipTo_Postal_Name_Middle Ecom_ShipTo_Postal_Name_Last Ecom_ShipTo_Postal_Name_Suffix Ecom_ShipTo_Postal_Street_Line1 Ecom_ShipTo_Postal_Street_Line2 Ecom_ShipTo_Postal_Street_Line3 Ecom_ShipTo_Postal_City Ecom_ShipTo_Postal_stateProv Ecom_ShipTo_Postal_Postalcode Ecom_ShipTo_Postal_CountryCode Ecom_ShipTo_Telecom_Phone_Number Ecom_ShipTo_Online_Email

Ecom_BillTo_Postal_Name_Prefix Ecom_BillTo_Postal_Name_First Ecom_BillTo_Postal_Name_Middle Ecom_BillTo_Postal_Name_Last Ecom_BillTo_Postal_Name_Suffix Ecom_BillTo_Postal_Street_Line1 Ecom_BillTo_Postal_Street_Line2 Ecom_BillTo_Postal_Street_Line3 Ecom_BillTo_Postal_City
Min Notes

$\begin{aligned} 4 & (1) \\ 15 & \\ 15 & (2) \\ 15 & \\ 4 & (3) \\ 20 & (4) \\ 20 & (4) \\ 20 & (4) \\ 22 & \\ 2 & (5) \\ 14 & (6) \\ 2 & (7) \\ 10 & (4) \\ 40 & (9) \\ & \\ 4 & (1) \\ 15 & \\ 15 & (2) \\ 15 & \\ 4 & (3) \\ 20 & (4) \\ 20 & (4) \\ 20 & (4) \\ 22 & \end{aligned}$

Informational [Page 7] 


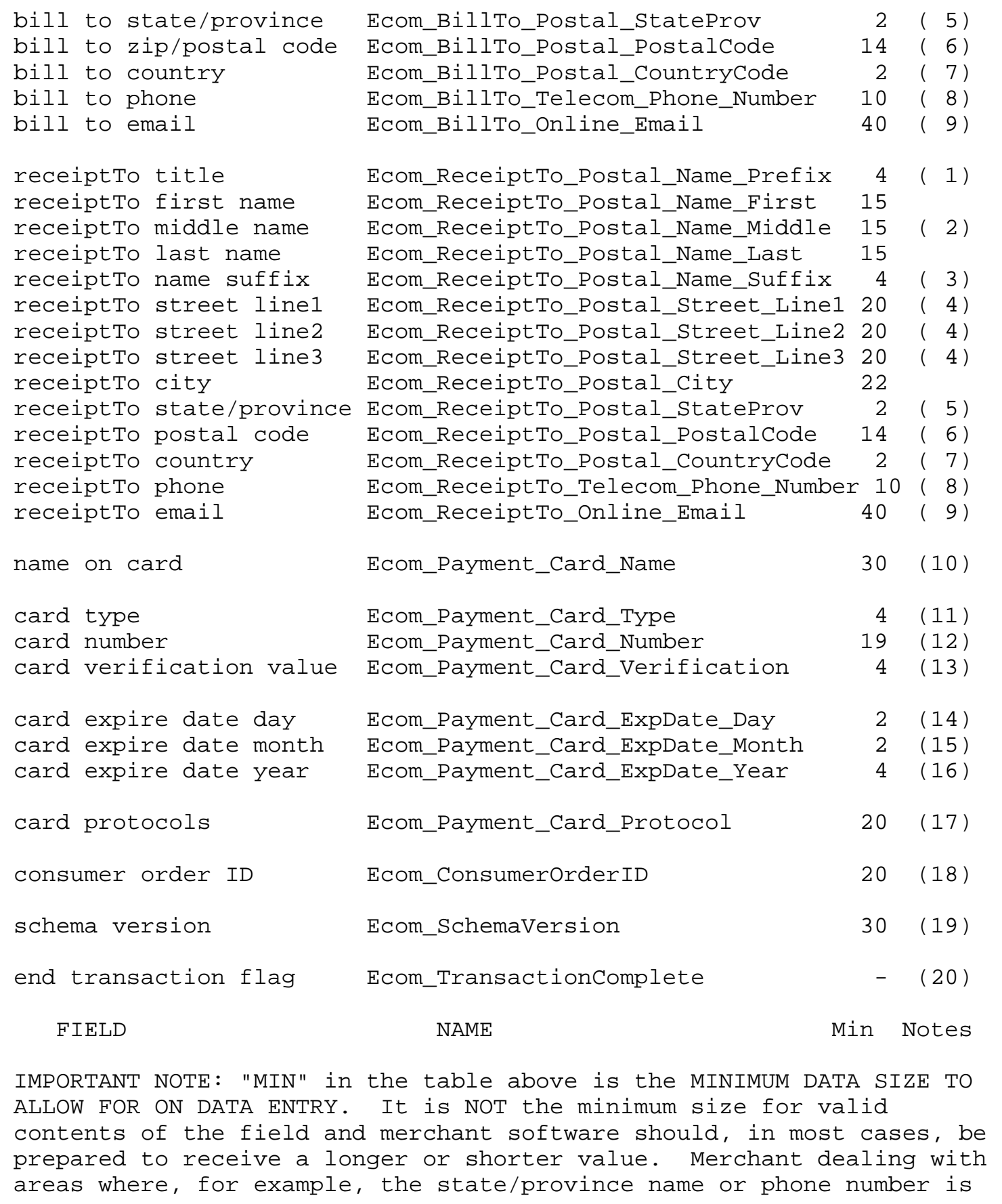


longer than the "Min" given below must obviously permit longer data entry. In some cases, however, there is a maximum size that makes sense and this is documented in a Note for the field.

4. End Notes

( 1) For example: Mr., Mrs., Ms., Dr. This field is commonly not used.

( 2) May also be used for middle initial

( 3) For example: Ph.D., Jr. (Junior), 3rd, Esq. (Esquire). This field is commonly not used.

(4) Address lines must be filled in the order line1, then line2, last line3.

( 5) 2 characters are the minimum for the US and Canada, other countries may require longer fields. For the Us use 2 character US Postal state abbreviation.

(6) Minimum field lengths for Postal Code will vary based on international market served. Use 5 character or $5+4$ ZIP for the US and 6 character postal code for Canada. The size given, 14, is believed to be the maximum required anywhere in the world.

( 7 ) Use [ISO 3166] standard two letter codes <http://www.din.de/gremien/nas/nabd/iso3166ma/codlstpl.html> for country names.

( 8) 10 digits are the minimum for numbers local to the North American Numbering Plan (<http://wWw.nanpa.com>: US, Canada and a number of smaller Caribbean and Pacific nations (but not Cuba)), other countries may require longer fields. Telephone numbers are complicated by differing international access codes, variant punctuation of area/city codes within countries, confusion caused by the fact that the international access code in the NANP region is usually the same as the "country code" for that area (1), etc. It will probably be necessary to use heuristics or human examination based on the telephone number and addresses given to figure out how to actually call a customer. It is recommend that an " $\mathrm{x}$ " be placed before extension numbers.

( 9) For example: jsmith@example.com

(10) The name of the cardholder. 
(11) Use the first 4 letters of the association name: American Express=AMER; Diners Club=DINE; Discover=DISC; JCB=JCB; Mastercard=MAST; Visa=VISA.

(12) Includes the check digit at end but no spaces or hyphens [ISO 7812]. The Min given, 19, is the longest number permitted under the ISO standard.

(13) An additional cardholder verification number printed on the card (but not embossed or recorded on the magnetic stripe) such as American Express' CIV, MasterCard's CVC2, and Visa's CVV2 values.

(14) The day of the month. Values: 1-31

(15) The month of the year. Jan - 1, Feb - 2, March - 3, etc.; Values: $1-12$

(16) The value in the wallet cell is always four digits, e.g., 1999, $2000,2001, \ldots$

(17) A space separated list of protocols available in connection with the specified card. Initial list of case insensitive tokens: none, set, \& setcert. "Set" indicates usable with SET protocol (i.e., is in a SET wallet) but does not have a SET certificate. "Setcert" indicates same but does have a set certificate. "None" indicates that automatic field fill is operating but there is no SET wallet or the card is not entered in any SET wallet.

(18) A unique order ID generated by the consumer software.

(19) URI indicating version of this set of fields. Usually a hidden field. Equal to "http://www.ecml.org/version/1.0" for this version.

(20) A flag to indicate that this web-page/aggregate is the final one for this transaction. Usually a hidden field.

5. Security Considerations

The information called for by many of these fields is sensitive and should be protected if being sent over the public Internet or through other channels where it can be observed. Mechanisms for such protection are not specified herein but channel encryption such as SSL/TLS [RFC 2246] or IPSec [RFC 2411] would be appropriate in many cases. 
User control over release of such information is needed to protect the user's privacy.

Any multi-web-page or other multi-aggregate field fill in or data provision mechanism should check for the Ecom_Transactioncomplete field and cease automated fill when it is encountered until fill is further authorized.

\section{References}

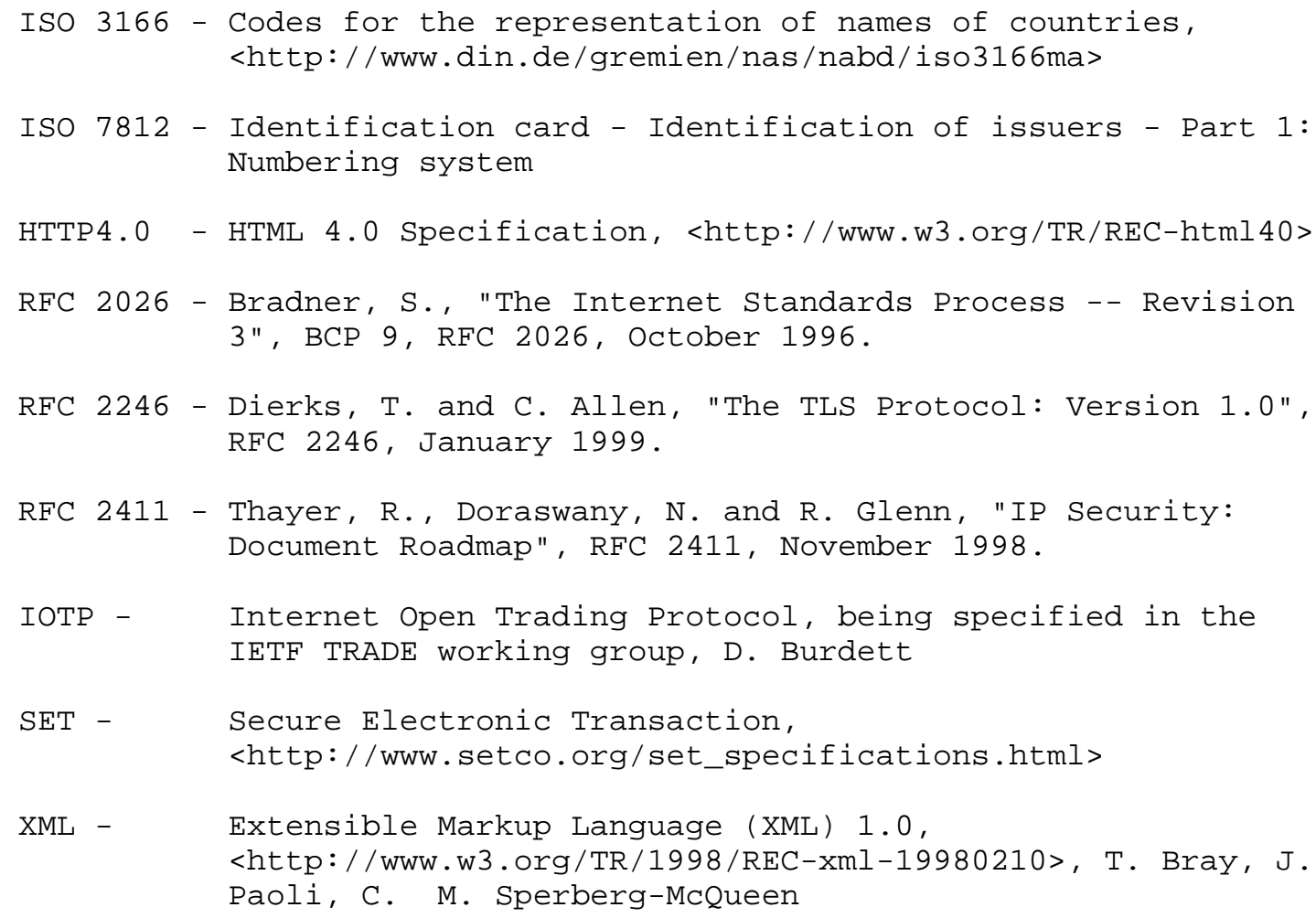




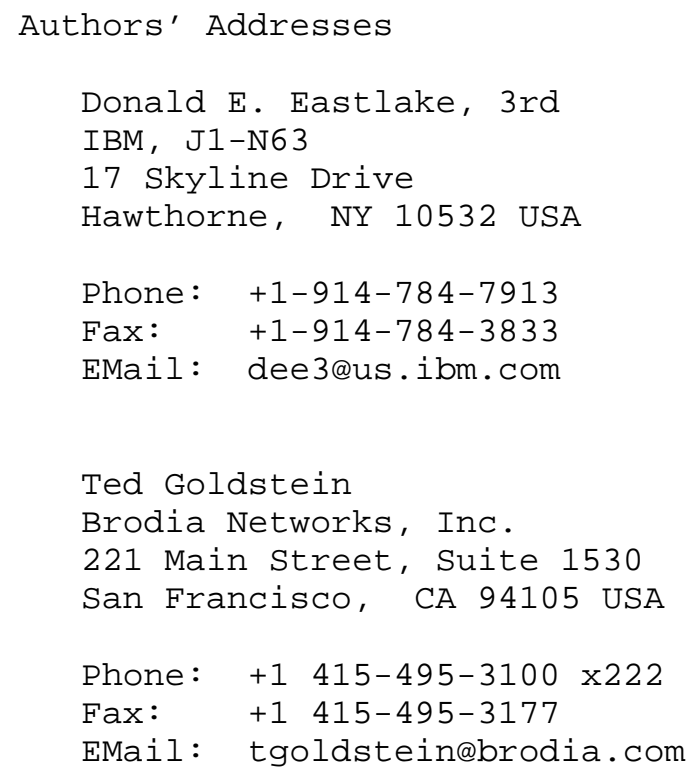


Full Copyright statement

Copyright (C) The Internet Society (1999). All Rights Reserved.

This document and translations of it may be copied and furnished to others, and derivative works that comment on or otherwise explain it or assist in its implementation may be prepared, copied, published and distributed, in whole or in part, without restriction of any kind, provided that the above copyright notice and this paragraph are included on all such copies and derivative works. However, this document itself may not be modified in any way, such as by removing the copyright notice or references to the Internet society or other Internet organizations, except as needed for the purpose of developing Internet standards in which case the procedures for copyrights defined in the Internet Standards process must be followed, or as required to translate it into languages other than English.

The limited permissions granted above are perpetual and will not be revoked by the Internet society or its successors or assigns.

This document and the information contained herein is provided on an "AS IS" basis and THE INTERNET SOCIETY AND THE INTERNET ENGINEERING TASK FORCE DISCLAIMS ALL WARRANTIES, EXPRESS OR IMPLIED, INCLUDING BUT NOT LIMITED TO ANY WARRANTY THAT THE USE OF THE INFORMATION HEREIN WILL NOT INFRINGE ANY RIGHTS OR ANY IMPLIED WARRANTIES OF MERCHANTABILITY OR FITNESS FOR A PARTICULAR PURPOSE.

Acknowledgement

Funding for the RFC Editor function is currently provided by the Internet society. 